\title{
A laceration of left internal mammary artery caused by an amateur golfer's swing
}

\author{
Deog-Gon Cho ${ }^{1,2}$, Yong-Jin Chang ${ }^{1,2}$, Kyu-Do Cho ${ }^{1,2}$ \\ ${ }^{1}$ Department of Thoracic and Cardiovascular Surgery, St. Vincent's Hospital, Suwon, Republic of Korea; ${ }^{2}$ College of Medicine, The Catholic \\ University of Korea, Seoul, Republic of Korea \\ Correspondence to: Yong-Jin Chang, MD. Department of Thoracic and Cardiovascular Surgery, St. Vincent's Hospital, Suwon, Republic of Korea; \\ College of Medicine, The Catholic University of Korea, Seoul, Republic of Korea. Email: tsmate@hanmail.net.
}

\begin{abstract}
We report the rare case of a patient presenting with a spontaneous laceration of left internal mammary artery (LIMA) after playing golf. The patient had no specific history except for cardiac surgery, and there were no results that caused bleeding on preoperative examination. A computed tomography (CT) scan of the chest demonstrated an anterior mediastinal hematoma and a left hemothorax with active extravasation close to LIMA. Through thoracotomy, hematoma evacuation and clipping for lacerated artery were performed. The patient was discharged in stable condition on the sixteenth postoperative day. This is the first reported case of a spontaneous laceration of internal mammary artery (IMA) after playing golf.
\end{abstract}

Keywords: Hemothorax; internal mammary artery (IMA); mediastinal hematoma; golf

Submitted Mar 25, 2017. Accepted for publication Sep 12, 2017.

doi: $10.21037 /$ jtd.2017.10.71

View this article at: http://dx.doi.org/10.21037/jtd.2017.10.71

\section{Introduction}

The laceration or rupture of the internal mammary artery (IMA) causes lethal massive bleeding. Most of the causes are blunt chest trauma, and few reports of spontaneous rupture have been reported. This study is thought to be the first report of laceration of the IMA associated with golfer's swing.

\section{Case presentation}

A 46-year-old man was admitted to our emergency center suffering from chest discomfort. He played golf before admission. The patient had a previous history of mitral valve replacement and Maze operation due to mitral stenosis and atrial fibrillation 2 years ago.

The patient's initial vital sign was stable. The laboratory data revealed a normal level except an elongated prothrombin time (international normalized ratio $=2.05$ ). A chest radiograph revealed a widened mediastinum (Figure 1A). A chest CT demonstrated an anterior mediastinal hematoma and a left hemothorax with active extravasation close to LIMA (Figure $1 B, C$ ). During the evaluation, the hemothorax worsened and the patient became hypotensive. However, the patient's vital sign recovered immediately after crystalloid infusion and remained stable thereafter.

Surgical exploration was undertaken through a posterolateral thoracotomy. An approximately 3,000 mL of fresh blood was encountered within the anterior mediastinum and thoracic cavity. A lacerated LIMA was identified as the source of bleeding at fourth intercostal space and was clipped (Figure $2 A$ ). The patient's postoperative course was uneventful. A chest CT on the tenth postoperative day revealed no evidence of contrast extravasation of LIMA (Figure $2 B$ ). The patient was discharged in stable condition on the sixteenth postoperative day. Follow-up examination at 2 years revealed the patient to be in good health, having experienced no untoward sequelae.

\section{Discussion}

IMA lies lateral to the sternum and therefore is particularly 

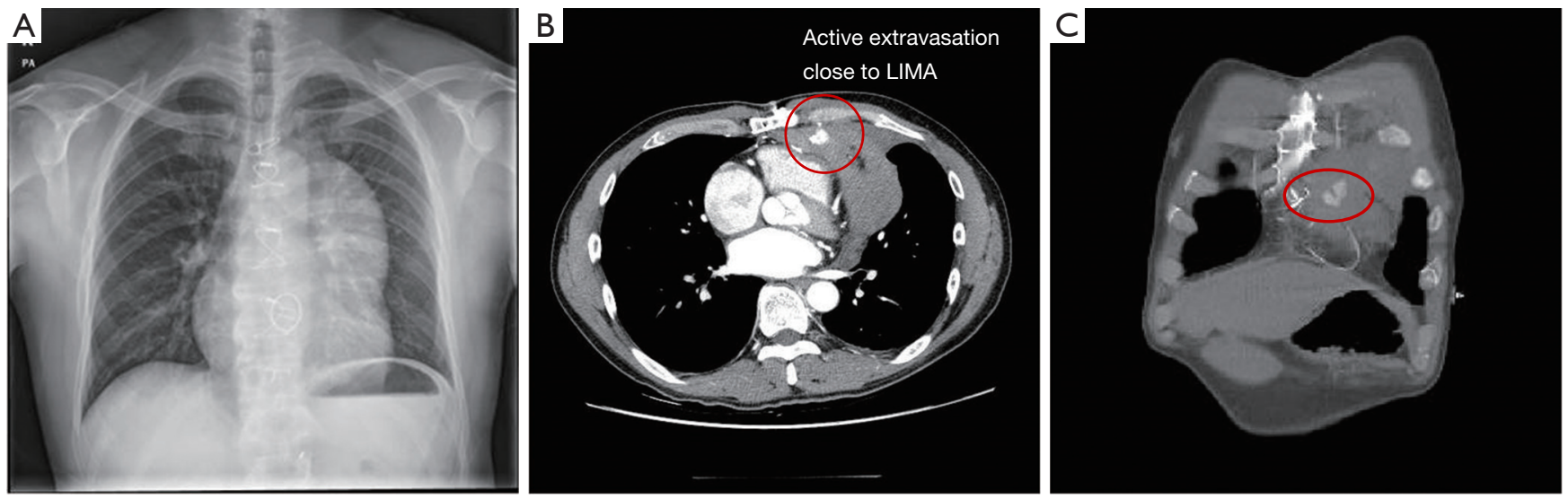

Figure 1 Initial chest CT images. (A) On plain X-ray, left anterior mediastinum widening is observed; (B,C) a chest CT demonstrated an anterior mediastinal hematoma and a left hemothorax with active extravasation close to LIMA (circle). CT, computed tomography; LIMA, left internal mammary artery.
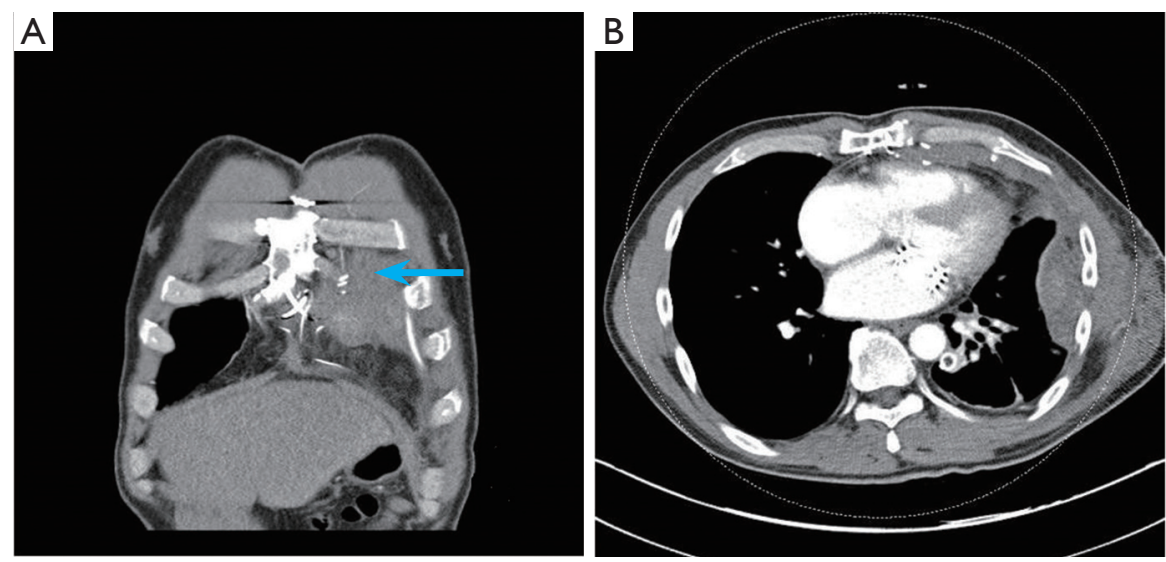

Figure 2 On a chest CT on the tenth postoperative day, a surgical clip was observed in the fourth intercostal space, and no further contrast extravasation was observed (arrow) (A,B). CT, computed tomography.

vulnerable to sternal and parasternal injury. There is a rich potential collateral network in the mediastinum and pericardium, which renders injury to this vessel prone to produce a mediastinal hematoma, pericardial tamponade, and massive hemothorax (1).

The cause of injury in this study is unclear. Two possible causes of injury may be considered in patient; the first is a rupture of false aneurysm that occurred after the closure of median sternotomy. Injury of an IMA during sternal closure is a well-known hazard. In addition, false aneurysm of an IMA, while rare, is known sequelae of sternal closure (2). However, no abnormal findings were observed during follow-up period after cardiac surgery and there was no suspicion of false aneurysm in surgical findings. Thus, the rupture of the false aneurysm could be ruled out.

The other is the occurrence of an injury due to the repetitive force applied to the rib in relation to the golf swing $(3,4)$. Golf is a sport that requires momentary strong impacts repeatedly. A proposed mechanism is that inexperienced golfers, who have poor form, repetitively strike the ground when they swing their club. This causes a forceful traction of the serratus anterior muscle, which consequently places stress on the ribs (5). The site of stress is most often the posterolateral ribs with four to six, and it is also known to occur in the anteromedial rib. We carefully estimate that laceration of LIMA was caused by a repetitive force on the anteromedial side of the rib during the golfer's 
swing.

In conclusion, we experienced a spontaneous laceration of the IMA, which seems to be related to golf swing. To our knowledge, it is a first case report of in literatures.

\section{Acknowledgements}

None.

\section{Footnote}

Conflicts of Interest: The authors have no conflicts of interest to declare.

Informed Consent: Written informed consent was obtained from the patient for publication of this case report and any accompanying images.

Cite this article as: Cho DG, Chang YJ, Cho KD. A laceration of left internal mammary artery caused by an amateur golfer's swing. J Thorac Dis 2017;9(11):E982-E984. doi: 10.21037/ jtd.2017.10.71

\section{References}

1. Whigham CJ Jr, Fisher RG, Goodman CJ, et al. Traumatic injury of the internal mammary artery: embolization versus surgical and nonoperative management. Emerg Radiol 2002;9:201-7.

2. Falconieri F, Raevsky E, Davies S, et al. Pseudoaneurysm of a branch of left internal mammary artery: a late and potentially fatal complication after redo-sternotomy. Interact Cardiovasc Thorac Surg 2015;20:866-7.

3. Lee AD. Golf-related stress fractures: a structured review of the literature. J Can Chiropr Assoc 2009;53:290-9.

4. Bugbee S. Rib stress fracture in a golfer. Curr Sports Med Rep 2010;9:40-2.

5. Goyal M, Kenney AJ 3rd, Hanelin LG. Golfer's rib stress fracture (Duffer's fracture). Scintigraphic appearance. Clin Nucl Med 1997;22:503-4. 